\title{
DESARROLLO DE UN PROTOTIPO DE CURSO VIRTUAL SOBRE SISTEMAS OPERATIVOS "PAVSO"
}

Por:

Oscar Alberto Gallardo Pérez' ,Liliana Esther Machuca Villegas²

\section{RESUMEN}

En los procesos de aprendizaje virtual no todo está en la tecnología; son fundamentales los componentes pedagógicos que giran alrededor de ellos y que sirven a la vez de base para su desarrollo. Consciente de esta realidad el Grupo de Investigación y Desarrollo de Ingeniería de Software (GIDIS) ha establecido unas Estrategias Metodológicas para el Aprendizaje en Aulas Virtuales de la UFPS que integradas con las Nuevas Tecnologías de Información y Comunicación fusionan la pedagogía con la tecnología en el desarrollo de un Prototipo para el Aprendizaje Virtual del Curso Sistemas Operativos denominado PAVSO que las integra y en el que se plasma un concepto de educación virtual adecuado a la infraestructura de comunicaciones existente en la región.

PAVSO se distingue por aplicar un enfoque pedagógico, enmarcado en el aprendizaje significativo constructivista, que orienta al estudiante hacia el aprendizaje autónomo, brindándole nuevas formas de aprendizaje y el desarrollo de competencias de tipo interpretativo, argumentativo, propositivo y comunicativo, rompiendo las barreras de espacio y tiempo que imponen las sesiones de clase tradicionales al hacer uso de las Nuevas Tecnologías de Información y Comunicación.

Palabras claves Aprendizaje virtual, Estrategias Metodológicas, Integración pedagogía - Tecnología, Sistemas Operativos.

\section{ABSTRACT}

In the processes of virtual learning everything is not in the technology; the pedagogical components that they turn around them are fundamental and who they serve simultaneously as base for his development. Conscious of this reality the Group of Investigation and Development of Engineering of Software (GIDIS) has established Methodologic Strategies for the Learning in Virtual Classrooms of the UFPS that integrated with the New Technologies of Information and Communication fuses pedagogy with the technology in the development of a Prototype for the Virtual Learning of the Course Operating Systems denominated PAVSO that Integrate them and which one shapes a concept of virtual education adapted to the existing infrastructure of communications in the region.

PAVSO is distinguished by apply a pedagogical approach, framed in a constructive significant learning, that it orients to the student towards the independent learning, offering him to new forms of learning and the development of competitions of interpretative, argue, propositive and communicative type, breaking the barriers of space and time that impose the sessions of traditional class when doing use of the New Technologies of Information and Communication.

PACS: 01.40.Di; 01.40.Gm; 07.05.Bx

\section{INTRODUCCIÓN}

El uso de Nuevas Tecnologías de la Información y Comunicación ha hecho que la educación superior busque como una alternativa la enseñanza y el aprendizaje en forma virtual [1-4]. Gracias a Internet, red de redes que permite la conexión entre diferentes computadores, los estudiantes podrán adaptar su trabajo a su tiempo y horario, ya que los cursos y servicios están disponibles las 24 horas, así mismo se reducen los costos por desplazamientos y se posibilita la formación de grupos geográficamente dispersos [5].

\footnotetext{
'Docente asociado. Departamento de Sistemas U.F.P.S., oagallar@bari.ufps.edu.co

2Ingeniero de Sistemas, Integrante Grupo de Investigación y Desarrollo de Ingeniería de Software "GIDIS",

lemachuc@bari.ufps.edu.co,
} 
También este tipo de aprendizaje se adapta a las necesidades y características de cada uno de sus usuarios, facilitando la interacción y el intercambio de conocimientos entre ellos. Sin embargo muchas veces toda la discusión gira alrededor de la tecnología dejando de lado los aspectos pedagógicos que subyacen en todos los procesos de aprendizaje; por esto, el Grupo de Investigación y Desarrollo de Ingeniería de Software en el Área de Informática Educativa de la UFPS, desarrolló el Diseño de Estrategias Metodológicas para el Aprendizaje en las Aulas Virtuales de la UFPS[6], y utilizando estas realizó un Prototipo para el Aprendizaje Virtual del curso presencial Sistemas Operativos[7], en el que se plasma un concepto de educación virtual, que propicia el hábito del uso de medios de información, que despierta el interés por el curso y estimula la formación mediante el autoaprendizaje y la disciplina hacia la investigación. Estos proyectos se han convertido en los pioneros del desarrollo de la Educación Virtual en la UFPS. Actualmente en el interior del Grupo de Investigación, se adelanta el Análisis de algunos Sistemas de Administración de Aprendizaje (LMS) de distribución gratuita[8-13], software necesario para gestionar los cursos, usuarios y herramientas de comunicación en los ambientes de formación virtual.

De igual manera se trabaja sobre otros prototipos para el aprendizaje virtual de las asignaturas Redes I y Redes Il del Programa de Ingeniería de Sistemas de la UFPS.

Con PAVSO y este tipo de proyectos se busca emprender el camino hacia un nuevo modelo de Enseñanza-Aprendizaje en la Universidad Francisco de Paula Santander, proporcionando herramientas para el aprendizaje virtual, cambiando la concepción de la enseñanza como sólo la transmisión de conocimientos y ampliando la posibilidad del aprendizaje autónomo.

\section{BASES METODOLÓGICAS}

El Prototipo para el Aprendizaje Virtual del curso Sistemas Operativos (PAVSO), involucra procesos de enseñanza-aprendizaje, basados en las Estrategias Metodológicas para el Aprendizaje en las Aulas Virtuales. Estas Estrategias Metodológicas buscan ser aplicadas a cursos que se desarrollen en la Web, en donde se utilice el correo electrónico, el chat, el foro como herramientas para la interacción entre los alumnos y el profesor[6]. Por tal razón, se hizo necesario integrarlas con la Metodología para el Desarrollo de Sitios Web[14]; enmarcándolas en una Metodología para la construcción de Multimedia y sus Elementos[15], de la cual se hizo la correspondiente adaptación con el fin de cumplir con los requisitos que el prototipo demanda, especialmente en los aspectos pedagógicos.

Para el desarrollo de la investigación se tomó como población 110 estudiantes de Ingeniería de Sistemas de la Universidad Francisco de Paula Santander que cursaron la asignatura Sistemas Operativos el segundo semestre de 2003, incluyendo los profesores responsables de ella[7].

El sitio Web que aloja a PAVSO[16], reúne el contenido teórico de los diferentes temas que corresponde al curso Sistemas Operativos, así como imágenes, animaciones, y actividades que permiten un mejor entendimiento del contenido. Este se encuentra alojado en el servidor bari de la Universidad Francisco de Paula Santander, el cual trabaja bajo plataforma Solaris, empleándose el puerto 10000 de éste para la implementación del chat.

PAVSO está dado para que el estudiante desarrolle competencias[17-18], entendiéndose éstas como el conocimiento que se posee y el uso que se hace de ese conocimiento al resolver una tarea en una situación especifica de acuerdo con unas necesidades y exigencias concretas; y como tal el producto ofrece los mecanismos necesarios para que el estudiante desarrolle competencias de tipo interpretativo, argumentativo, comunicativo y propositivo, a través de las cuales pueda construir su aprendizaje de una 
manera autónoma, que le enriquezca y le permita desenvolverse en el curso en cuestión.

\section{Análisis Estructural del Aprendizaje}

Las estrategias pedagógicas empleadas en el desarrollo de PAVSO están fundamentadas en el Aprendizaje Significativo Constructivista[19-22].

En el Aprendizaje Constructivista, el alumno es el responsable de su propio proceso de aprendizaje. Es él quien construye el conocimiento y nadie puede sustituirle en esa tarea. El alumno no es sólo activo cuando manipula, explora, descubre o inventa, sino también cuando lee o escucha las explicaciones del facilitador[6].

El Aprendizaje Significativo surge cuando el alumno, como constructor de su propio conocimiento relaciona los conceptos a aprender y les da sentido a partir de la estructura conceptual que ya posee. Construye nuevos conocimientos a partir de los conocimientos que ha adquirido anteriormente. Este puede ser por descubrimiento o por recepción. Pero además construye su propio conocimiento porque quiere y está interesado en ello. El Aprendizaje Significativo algunas veces se construye al relacionar los conceptos nuevos con los conceptos que ya posee y otras al relacionar los conceptos nuevos con experiencias previas[6].

\section{Marco Estructural del Conocimiento}

En el marco estructural del conocimiento, se relacionan los diferentes temas y subtemas del curso Sistemas Operativos definidos por el Programa de Ingeniería de Sistemas de la UFPS, éstos se representan a través de nodos y arcos[7, 23,24].

\section{Estrategia Instruccional}

La estrategia instruccional a seguir para lograr los objetivos de aprendizaje, está estructurada en cuatro fases: Retomar conceptos previos, construir nuevos conceptos, fortalecer los conceptos y evaluar competencias. En cada tema se desarrolla una serie de actividades como lecturas, pruebas, crucigramas, juegos, analogías, ejercicios, explicaciones, análisis de imágenes, simulaciones y animaciones. Estas constan de sugerencias que le permiten al estudiante constatar la exactitud de sus respuestas. En algunos casos no encontrará "soluciones" ya que se trata simplemente de reflexionar sobre la experiencia acumulada hasta el momento y plasmar tales reflexiones en un documento.

El propósito de la fase uno (Retomar conceptos previos), es propiciar una primera revisión sistemática de algunos conceptos fundamentales sobre el tema a través del desarrollo de una serie de actividades que le son familiares al estudiante, y que permiten iniciar el estudio de otros temas que presentan una mayor complejidad y que requieren de un nivel más desarrollado de abstracción y habilidades más específicas. Se trata de presentar al estudiante una serie de esquemas, figuras, imágenes, simulaciones, situaciones, ejercicios con el fin de que pueda asociarlas o relacionarlas con el nuevo tema, para que partiendo de allí empiece a formarse una concepción del contenido.

En la fase dos (construir nuevos conceptos), las actividades están orientadas hacia el aprendizaje del nuevo tema, los conceptos se van presentando acompañados de ilustraciones, animaciones, ejemplos y situaciones que permiten ampliar su explicación. También se resuelven ejercicios paso a paso para que así el estudiante, integre el concepto con práctica y pueda aplicarlos más adelante en los ejercicios propuestos y más importante aún en su vida profesional.

El objetivo de la tercera fase es fortalecer los conceptos (Confronta los conceptos e ideas previas con los conceptos nuevos, que se busca aprendan). Esta fase se caracteriza por integrar actividades como animaciones, crucigramas, lecturas y ejercicios, que permiten el afianzamiento de los mismos. 
En la fase cuatro (evaluar competencias), se enfrenta al estudiante a una serie de retos que le permiten desarrollar competencias comunicativa, interpretativa y propositiva. Es importante resaltar que el producto facilita al estudiante las herramientas necesarias para que pueda desarrollar actividades de autoevaluación que le permiten establecer el grado de aprendizaje de cada uno de los temas tratados.

El desarrollo de cada una de las fases gira alrededor del planteamiento de preguntas. Estas son una actividad típicamente humana y uno de los puntos de apoyo inevitables en las actitudes creativas. Sin lugar a dudas, es un importante recurso por el que se puede orientar el aprendizaje de los alumnos hacia la dirección establecida. Con las preguntas se predefine el tipo de operación mental que se desea explicitar.

Con las animaciones y las actividades que se incluyen se pretende crear un conjunto rico de experiencias de modo que facilite a los estudiantes la construcción de nuevos conceptos y la participación activa en su proceso de aprendizaje del curso Sistemas Operativos, en lugar de ser un espectador pasivo. El hecho de relacionar el nuevo conocimiento con conocimientos previos o con situaciones de la vida cotidiana, facilitan la asimilación y el aprendizaje ya que el estudiante normalmente recuerda este nuevo conocimiento a partir de este tipo de asociación.

\section{Pruebas Instruccionales}

Las pruebas instruccionales para evaluar el logro de los objetivos instruccionales específicos y los objetivos definidos en cada uno de los temas del curso, se organizaron de la siguiente manera:

- Respuesta objetiva (ítems cerrado)

a) Falso / Verdadero

b) Completación o llenar espacio en blanco

c) Apareamiento o correspondencia

d) Selección múltiple

e) Análisis de relación
- Respuesta libre (ítem abierto)

Expresar con sus propias palabras

- Respuesta de ensayo o composición

Herramientas utilizadas para el desarrollo de PAVSO

PAVSO se desarrolló empleando las siguientes herramientas:

- Herramientas para la edición de texto: Microsoft Word, WordPad y Bloc de notas de Microsoft Windows Me.

- Herramienta para el diseño de páginas Web: Macromedia Dreamweaber MX versión prueba y HTML 4.01.

- Herramientas para la creación y edición de imágenes: CorelDraw 10, Corel PhotoPaint 10 y Paint de Microsoft Windows Me.

- Herramienta para la captura de imágenes: Corel PhotoPaint 10.

- Herramienta para la creación de animaciones: Macromedia Flash 6 versión prueba.

- Herramienta para la creación de Gifs animados: Microsoft GIF Animator.

- Herramienta Orientada a Objetos para la creación de applets y chat: JAVA 1.4.0.

- Herramienta para desarrollar ejercicios interactivos: Hot Potatoes versión 5, Rayuela. Software gratuito.

- IDE (Entorno de Desarrollo Integrado de Java): JCreator versión 2.5 software gratuito.

- Visualizador de páginas Web: Internet Explorer 5.0.

\section{DISEÑO}

En esta etapa se especificó la presentación del sitio Web desarrollando la interfase para permitir al usuario llegar a la información requerida a través de los mapas de navegación $[6,23,24]$. Además se integraron las Estrategias Metodológicas con el fin de estructurar el ciclo de aprendizaje virtual del Curso Sistemas Operativos. 
Para la realización de PAVSO se tuvo en cuenta la Metodología para el desarrollo de sitios web, pues en ella se especifican requerimientos y recomendaciones necesarias para la construcción de un sitio Web, a su vez se aplicaron los conocimientos y habilidades en lo que respecta al diseño gráfico y empleo adecuado de los recursos didácticos con el fin de brindar una interfaz agradable y rutas de navegación óptimas.

El diseño de la interfaz presenta diferentes escenas a través de las cuales los usuarios de PAVSO pueden desenvolverse y desarrollar todas las actividades que se orientan hacia el aprendizaje del curso. Algunas de ellas se reflejan en las siguientes Figuras.

Figura 1. Página de Inicio de PAVSO. A través de ella los usuarios pueden ingresar a cualquier unidad del curso, enviar un correo o conectarse al chat.

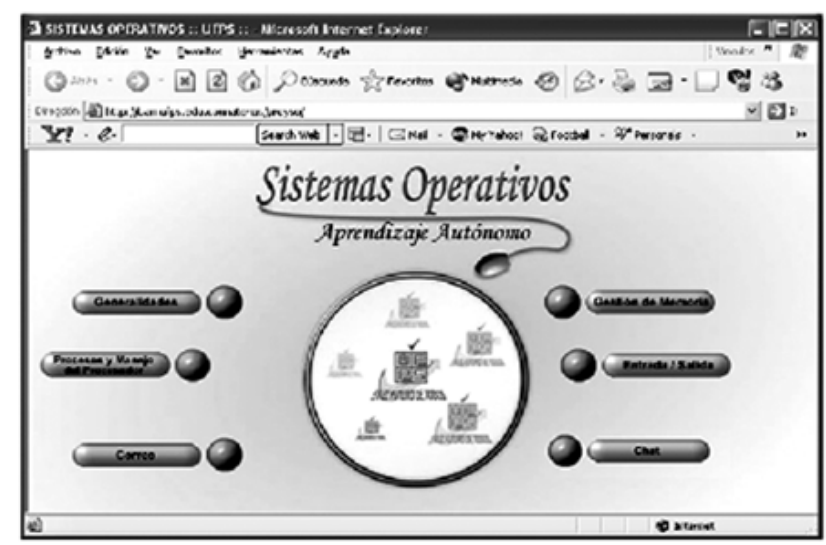

Figura 2. Presentación de las actividades. Para el desarrollo del contenido se han utilizado una serie de actividades que se caracterizan por estar identificadas con íconos (imágenes). Algunos de ellos representarán un vínculo que lo llevarán a una animación o interactividad.

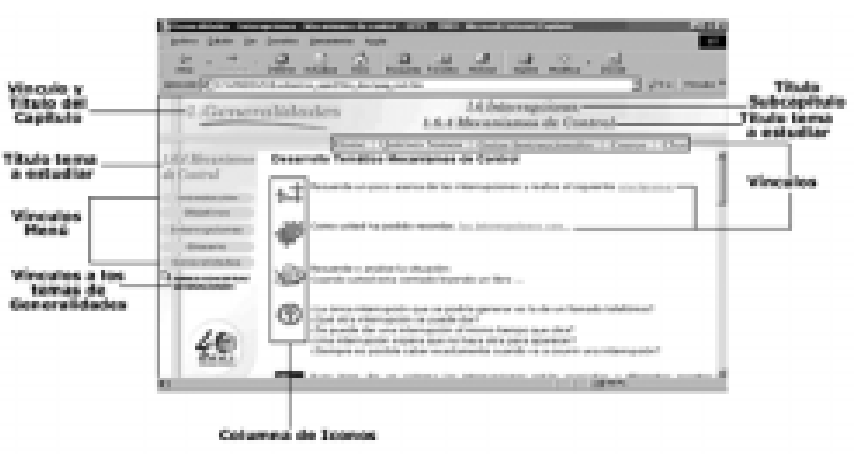

Figura 3. Al ejecutar la animación se refleja el proceso de la traducción de una dirección virtual utilizando el Mecanismo de Traducción de direcciones por Paginación

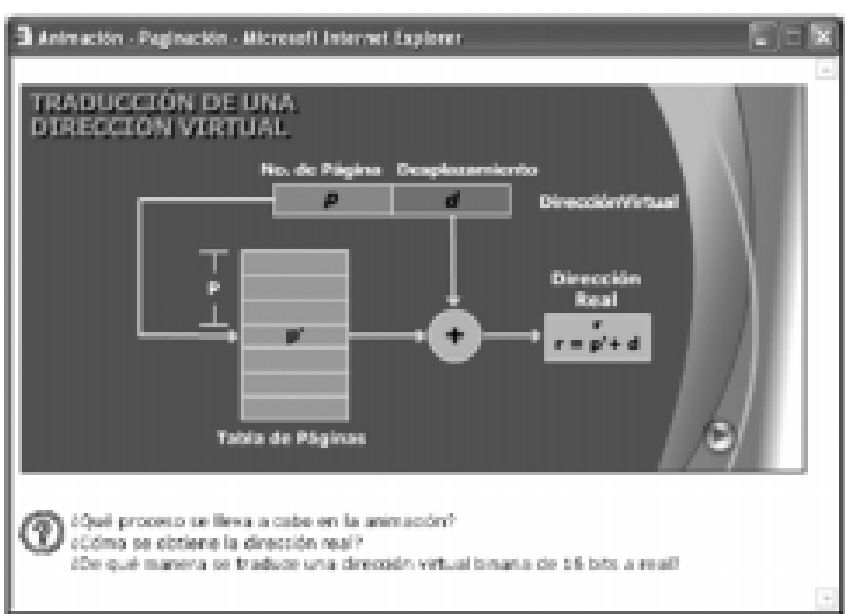

\section{PRUEBAS}

PAVSO estuvo expuesto a pruebas de tipo alfa y beta, con el fin de evaluar su funcionalidad [7]. Estas consisten en probar la herramienta en forma interna dentro del equipo de trabajo (pruebas alfa) y con los usuarios finales en un ambiente real sin incluir personas involucradas en el desarrollo del proyecto (pruebas beta). Durante el desarrollo de éstas se encontraron fallas relacionadas con la navegación, sintaxis y en algunas actividades de aprendizaje. Así mismo, una vez corregidos estos inconvenientes se aplicó una encuesta a los estudiantes que interactuaron con el prototipo y una entrevista a los docentes del área; con ellas se obtuvo una retroalimentación que fue considerada para el refinamiento final del proyecto.

En la actualidad PAVSO es utilizado con muy buena aceptación por los estudiantes del curso Sistemas Operativos del Programa de Ingeniería de Sistemas de la UFPS. Así mismo, se manifiesta lo útil y didáctica que es la herramienta en el proceso de aprendizaje. 


\section{CONCLUSIONES}

Es fundamental manejar un enfoque pedagógico en los proyectos que se orientan hacia la educación virtual, pues es la pieza angular para el desarrollo de los mismos, ya que permite estructurar el aprendizaje, indicando la metodología a seguir y el tipo de actividades que se deben proponer al alumno, enmarcándolas dentro de las herramientas tecnológicas disponibles en el medio.

Es de destacar la utilidad de las Estrategias Metodológicas para el Aprendizaje en Aulas Virtuales de la UFPS en el desarrollo de PAVSO, ya que constituyen la base pedagógica del proyecto y gracias a ellas, se orientó la evolución del aprendizaje del curso.

La utilización de las Estrategias Metodológicas en el prototipo demostró que se puede aplicar un enfoque pedagógico a los contenidos y cursos que se desarrollen en la Web.

\section{BIBLIOGRAFÍA}

1. Henao, O. (2002). La Enseñanza Virtual en la Educación Superior. (Primera Edición). Bogotá. Instituto Colombiano para el Fomento de la Educación Superior ICFES.

2. Bello, R. Educación Virtual Aulas sin paredes. http:/ /www.educar.org/articulos/educacionvirtual.asp

3. Candia, A. La educación Virtual: ¿̇Una alternativa a la educación tradicional? . http://www.utdt.edu/ eduforum/ensayo 10.htm

4. Olarte, L. Empezó el boom del e-learning. Artículo. h†tp://www.elearningworkshops.com / modules. php? name $=$ News\&file $=$ article $\&$ sid $=1$ $42 \&$ mode $=$ \&order $=0 \&$ thold $=0$
5. Qs-media. Ventajas del elearning. http:// www.qsmedia.es/elearning/elr_ventajas.cfm? subop $=2$

6. Galvis, L. \& Reyes, L. (2001). Diseño de estrategias Metodológicas para el Aprendizaje en las Aulas Virtuales de la Universidad Francisco de Paula Santander. Memoria para optar el Título de Ingeniero de Sistemas, Programa de Ingeniería de Sistemas, Universidad Francisco de Paula Santander, San José de Cúcuta, Colombia.

7. Machuca, L. \& Mora, N. (2003). Prototipo para el Aprendizaje Virtual de la Unidad de Gestión de Memoria de la Asignatura Sistemas Operativos del Plan de Estudios de Ingeniería de Sistemas de la UFPS. Memoria para optar el Título de Ingeniero de Sistemas, Programa de Ingeniería de Sistemas, Universidad Francisco de Paula Santander, San José de Cúcuta, Colombia.

8. Cordero, R. (2002). LMS: Una herramienta de difusión de conocimiento. (Volumen 1, No3). Aura Interactiva. www.aurainteractiva.com

9. Delgado, S. (2003). Elearning. Análisis de Plataformas Gratuitas. Proyecto Fin de Carrera Ingeniería Informática, Universidad de Valencia, España.

10. Sistema de Administración de Aprendizaje "MOODLE". www.moodle.org

11. Sistema de Administración de Aprendizaje "ATUTOR". www.atutor.ca

12. Sistema de Administración de Aprendizaje "DOKEOS". http://www.dokeos.com

13. Sistema de Administración de Aprendizaje "BCSW/ BSCL". http://bscl.gmd.de y http://bscw.gmd.de

14. Gallardo, O. (2001). Guías Generales para el Desarrollo de Sitios Web. San José de Cúcuta. 
15. Calderón, L. \& Contreras, D. E. (2002). Diseño y elaboración de una aplicación multimedia sobre una metodología para la construcción de una multimedia y sus elementos. Memoria para optar el Título de Ingeniero de Sistemas, Programa de Ingeniería de Sistemas, Universidad Francisco de Paula Santander, San José de Cúcuta, Colombia.

16. Dirección del sitio web del Prototipo para el Aprendizaje Virtual del Curso Sistemas Operativos http://bari.ufps.edu.co/materias/proyso/

17. BOGOYA, D. (2000). Competencias y proyectos pedagógicos. Santafé de Bogotá, D.C. Colombia. Ed. Unilobos.

18. TORRADO, M. (2000). Competencias y proyectos pedagógicos. Educar para el desarrollo de las competencias: Una propuesta para reflexionar. Universidad Nacional de Colombia. Santafé de Bogotá, D.C. Colombia.

19. Portal Educativo Argentino para Hispanoamérica y el mundo. Teoría del aprendizaje significativo por David Ausubel.

http://www.educainformatica.com.ar/docentes/ tuarticulo/educacion/ausubel/index.htm

20. Lahitte, L. ¿̇Qué es el constructivismo?. Portal Educativo Argentino para Hispanoamérica y el mundo. http://www.educainformatica.com.ar/docentes/ tuarticulo/educacion/constructiv/index.html

21. Teoría del Aprendizaje Significativo de David Ausubel. Trabajo enviado por: W. Palomino N. http://www.monografias.com/ trabajos6/apsi/apsi.shtml

22. Ibarra, A. (2001). Diplomado en Docencia y Didáctica: Pedagogía Constructivista. Universidad Francisco de Paula Santander, Facultad de Educación, Artes y Humanidades. San José de Cúcuta.
23. González, L. \& Sanjuán, L. (2004). Prototipo para el Aprendizaje Virtual de las Unidades de Generalidades y Procesos y Manejo de Procesador de la Materia Sistemas Operativos del Plan de Estudios de Ingeniería de Sistemas de la UFPS. Memoria para optar el Título de Ingeniero de Sistemas, Programa de Ingeniería de Sistemas, Universidad Francisco de Paula Santander, San José de Cúcuta, Colombia.

24. Pacheco, S. \& Pérez, S. (2004). Prototipo para el Aprendizaje Virtual de la Unidad de Entrada y Salida de la Materia Sistemas Operativos del Plan de Estudios de Ingeniería de Sistemas de la UFPS. Memoria para optar el Título de Ingeniero de Sistemas, Programa de Ingeniería de Sistemas, Universidad Francisco de Paula Santander, San José de Cúcuta, Colombia.

Recibido: Febrero 7 de 2005

Aceptado: Junio 13 de 2005 